\title{
COLLECTION OF LISTERIA MONOCYTOGENES ISOLATES FROM MILK, DAIRY PRODUCTS AND FOOD PROCESSING ENVIRONMENTS IN SLOVAKIA FOR THE PURPOSES OF EUROPEAN MOLECULAR DATABASE
}

\author{
Kubicová, Z., Filipová, M., Jurovčíková, J., Cabanová, L. \\ State Veterinary and Food Institute, Veterinary and Food Institute in Dolný Kubín \\ Jánoškova 1611/58, 02601 Dolný Kubín \\ Slovakia \\ miriam.filipova@svpu.sk
}

\section{ABSTRACT}

The molecular typing of Listeria monocytogenes isolates is an important tool for monitoring the spread of the strains in food chains, providing evidence for epidemiological investigations and for the detection of outbreaks. The demand of European typing data centralization, collection and sharing stimulated the generation of "EURL L. monocytogenes Database (EURL Lm DB)" in 2012 led by the European Union Reference Laboratory (EURL) for L. monocytogenes (ANSES Maisons-Alfort Laboratory for Food Safety, France) in close collaboration with Applied Maths. This database includes the typing results and epidemiological information on strains isolated from food, environmental or animal samples and it is in connection with human strains database TESSy (The European Surveillance System) led by the ECDC (European Centre for Disease Prevention and Control). In total 147 L. monocytogenes isolates were examined by PFGE (pulsed field gel electrophoresis) in 2014-2015 in VFI Dolny Kubin from different sources. Nearly half (68) of the 147 isolates in the national Slovak database came from milk or dairy products samples and the related manufacturing environment. In this work, 68 isolates associated with milk were selected and divided into 27 clusters (95\% similarity level) after combined comparison analysis (AscI and ApaI) by BioNumerics 6.6 software. Eight clusters included three or more similar PFGE profiles.

Key words: database; Listeria monocytogenes; molecular typing; PFGE

\section{INTRODUCTION}

The molecular typing of bacterial DNA by different methods (pulsed field gel electrophoresis - PFGE, multi locus variable-number tandem repeat analysis - MLVA, whole genome sequencing - WGS) has become a standard process of pathogenic foodborne bacteria characterization. The PFGE typing of foodborne microorganisms is very important for surveillance purposes, especially monitoring the spread of strains in food chains, ensuring evidence for 
epidemiological investigations and revelation of national or international outbreaks. PFGE is regarded as the "gold standard" among typing methods [6].

A surveillance network based on PFGE method including food and clinical isolates (PulseNet) has been used in USA and Canada for many years and it helped to detect numerous outbreaks [1,2,3]. PulseNet Europe was created with the same aim in 2003 [8], but it was cancelled in 2006 due to lack of funding [9]. In 2012 the European Centre for Disease Prevention and Control - ECDC developed a pilot Molecular Surveillance System (MSS) as a component of The European Surveillance System - TESSy. The scope of this databasing system is to share epidemiological information and molecular typing data on L.monocytogenes strains isolated in cases of human disease [10]. However, there was a constant need to collect and share information on European L. monocytogenes isolates sourced from food, animal and environmental samples. This need was satisfied in 2012 by the creation of "EURL L. monocytogenes Database (EURL Lm DB)" led by European Union Reference Laboratory (EURL) for Listeria monocytogenes (ANSES Maisons-Alfort Laboratory for Food Safety, France).The National Reference Laboratories (NRLs) across Europe can store and also share their molecular and epidemiological data on L. monocytogenes strains isolated from food, animal and environmental samples. The principle of data sharing is based on internet communication using BioNumerics software (Applied Maths Saint-Martens-Latem, Belgium) [5].

\section{MATERIALS AND METHODS}

In sum, 147 L.monocytogenes isolates from different sources were typed by PFGE in NRL for L. monocytogenes at the Veterinary and Food Institute (VFI) in Dolný Kubín during 2014-2015. However, this study was focused on 68 isolates from milk, dairy products and related food processing environments. The isolates originated in official control samples or from samples of producer's self-control. All L. monocytogenes isolates were analysed according to the EURL protocol [7]. Agarose plugs with bacterial DNA were prepared and restriction enzymes AscI and ApaI (Thermo Scientific, USA) were used for PFGE profiles production. Electrophoresis was performed in $1 \%$ agarose (SeaKem Gold Agarose, Lonza, USA) on a CHEF Mapper ${ }^{\circledast}$ XA (BioRad, USA). Salmonella Braenderup H9812 DNA cleaved with XbaI enzyme (Thermo Scientific, USA) was used as a reference system. The molecular profiles obtained by the PFGE procedures were analysed by BioNumerics v6.6 software (Applied Maths, Belgium) using the Dice coefficient and unweighted pair group method (UPGMA) with arithmetic mean analysis, with "optimization" and "tolerance" settings of $1 \%$. The inclusion of PFGE profiles into one group - cluster - was done according to $95 \%$ similarities among ApaI/AscI compared profiles.

\section{RESULTS}

From 2014 to 2015, a total of 147 L. monocytogenes from different sources were examined by PFGE in NRL for L. monocytogenes at VFI Dolný Kubín. These milk-associated isolates (milk, dairy products and related manufacture environments) formed nearly half (46.26\%) of all investigated isolates. The combined clustering analysis of $68 \mathrm{ApaI} /$ AscI profiles resulted in 27 clusters with $95 \%$ similarity.

Only 8 of the 27 clusters contained 3 or more identical, similar or nearly similar PFGE profiles (Fig. 1, Table 1). All 8 clusters were comprised of 44 isolates in total. The biggest cluster IV was composed of L. monocytogenes isolates from one specific source - sheep (sheep milk - 2 samples, products from sheep milk -8 samples and specific sheep milk processing environments -2 samples), and from one manufacturer. Conventional serotyping classified all 12 isolates into $1 / 2 \mathrm{a}$ serogroup. The epidemiological data of the other 7 clusters (I.-II., V.-VIII.) were also investigated in detail, but there was no confirmation of either the presence of isolates from the same source and time, or the connection with one manufacturer.

The remaining 19 clusters were formed only by one or two isolates per cluster and were comprised of 24 isolates in total (data not shown).

\section{DISCUSSION}

A surveillance of the L.monocytogenes spread in food chains is an essential part in preventing disease and monitoring public health issues. The surveillance may include sampling of food or environments for the presence or absence of the organisms which may contribute to the identification of the risky food batches or identify colonization of 


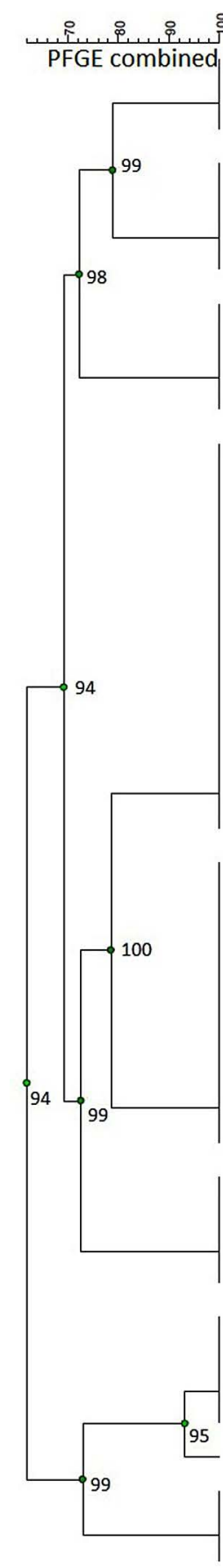

PFGE-Ascl
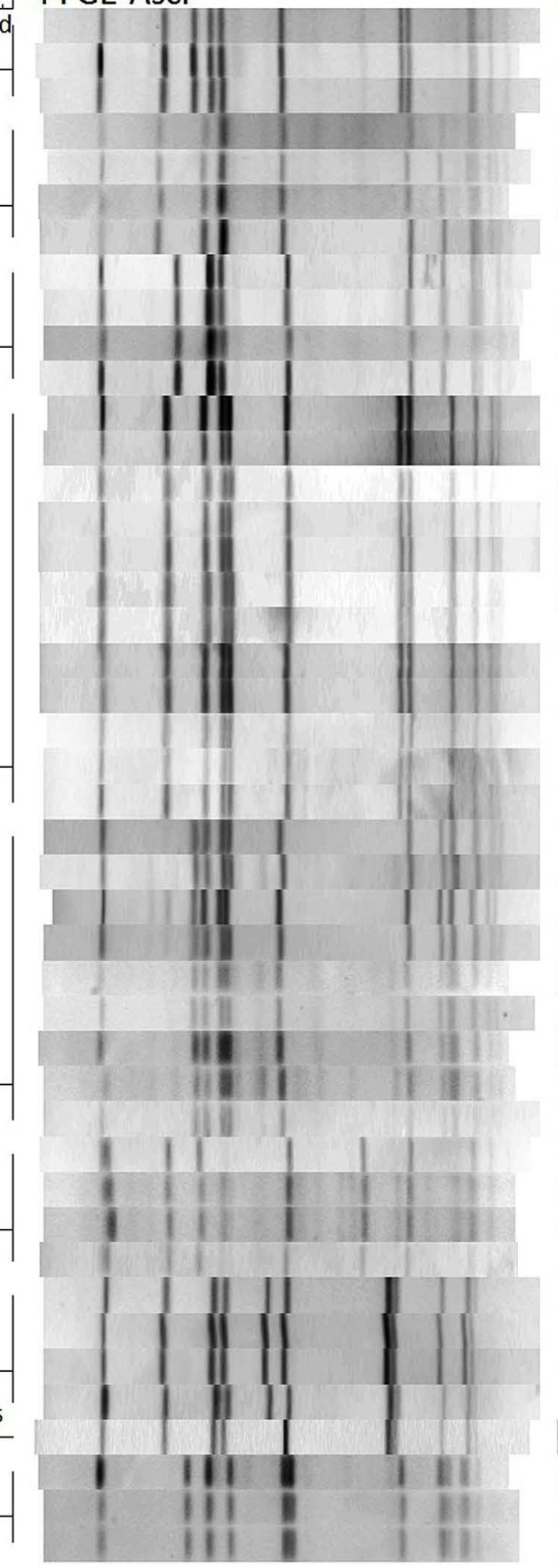

Fig 1. Similarity dendrogram of 8 PFGE clusters identified in the positive samples of milk, dairy products and related food processing environments

Combined analysis of AscI and ApaI profiles of 44 L. monocytogenes strains resulted in 8 clusters (I.-VIII.). Cluster IV was composed of 12 L. monocyogenes samples isolated from a specific source and one manufacturer. Despite the profiles similarity, all of the other clusters did not show a connection either with the source and time of sampling or with one producer
Sample ID Cluster

IV.

v.

VI.

VII.

VIII.

III.

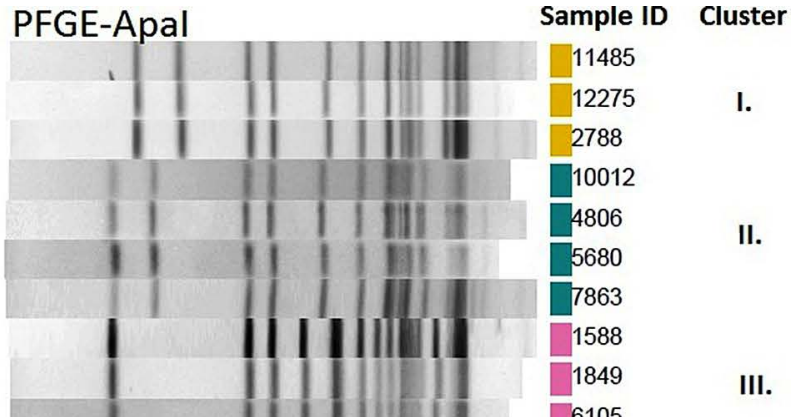


Table 1. Detailed epidemiological data concerning the samples of 8 PFGE clusters

\begin{tabular}{|c|c|c|c|c|c|}
\hline Cluster & Sample ID & $\begin{array}{c}\text { Conventional } \\
\text { serotype }\end{array}$ & Source & Sample category & $\begin{array}{l}\text { Sampling } \\
\text { date }\end{array}$ \\
\hline \multirow{3}{*}{ I. } & 11485 & $1 / 2 a$ & Sheep & Cheese category not specified & 2014-09-30 \\
\hline & 12275 & $1 / 2 a$ & Sheep & Soft cheese & 2014-10-17 \\
\hline & 2788 & $1 / 2 a$ & Sheep & Cheese category not specified & 2014-03-28 \\
\hline \multirow{4}{*}{ II. } & 10012 & $1 / 2 a$ & - & Food processing environment & $2014-08-26$ \\
\hline & 4806 & $1 / 2 a$ & Sheep & Cheese category not specified & 2014-05-13 \\
\hline & 5680 & $1 / 2 a$ & Sheep & Cheese category not specified & $2024-05-26$ \\
\hline & 7863 & $1 / 2 a$ & Sheep & Cheese category not specified & 2015-07-16 \\
\hline \multirow{4}{*}{ III. } & 1588 & $1 / 2 a$ & Bovine & Semi soft cheese & 2015-03-05 \\
\hline & 1849 & $1 / 2 a$ & Sheep & Cheese category not specified & 2014-03-07 \\
\hline & 6105 & $1 / 2 a$ & - & Food processing environment & 2014-06-03 \\
\hline & 978 & $1 / 2 a$ & Bovine & Cheese category not specified & $2015-02-17$ \\
\hline \multirow{12}{*}{ IV. } & 6282 & $1 / 2 a$ & Sheep & Soft cheese & 2015-06-16 \\
\hline & 6399 & $1 / 2 a$ & Sheep & Cheese category not specified & 2015-06-17 \\
\hline & 6644 & $1 / 2 a$ & Sheep & Soft cheese & $2015-06-23$ \\
\hline & 6818 & $1 / 2 a$ & - & Food processing environment & $2015-06-24$ \\
\hline & 6820 & $1 / 2 a$ & - & Food processing environment & 2015-06-24 \\
\hline & 6825 & $1 / 2 a$ & Sheep & Milk & $2015-06-24$ \\
\hline & 7180 & $1 / 2 a$ & Sheep & Semi soft cheese & 2015-07-01 \\
\hline & 8156 & $1 / 2 a$ & Sheep & Semi soft cheese & $2015-07-23$ \\
\hline & 8157 & $1 / 2 a$ & Sheep & Soft cheese & $2015-07-23$ \\
\hline & 8670 & $1 / 2 a$ & Sheep & Cheese category not specified & 2015-07-29 \\
\hline & 9575 & $1 / 2 a$ & Sheep & Soft cheese & $2015-08-21$ \\
\hline & 9578 & $1 / 2 a$ & Sheep & Milk & $2015-08-21$ \\
\hline \multirow{9}{*}{ V. } & 13326 & $1 / 2 a$ & Sheep & Soft cheese & 2014-11-10 \\
\hline & 14578 & $1 / 2 a$ & Sheep & Soft cheese & 2014-12-11 \\
\hline & 3266 & $1 / 2 a$ & Sheep & Cheese category not specified & 2015-04-16 \\
\hline & 4589 & $1 / 2 a$ & Sheep & Soft cheese & 2014-05-07 \\
\hline & 5247 & $1 / 2 a$ & Sheep & Soft cheese & $2014-05-20$ \\
\hline & 5893 & $1 / 2 a$ & Bovine & Semi soft cheese & $2011-05-28$ \\
\hline & 5894 & $1 / 2 a$ & Sheep & Milk & 2014-05-26 \\
\hline & 6215 & $1 / 2 a$ & Sheep & Soft cheese & 2014-06-05 \\
\hline & 9721 & $1 / 2 a$ & Bovine & Other dairy product & 2014-08-25 \\
\hline \multirow{4}{*}{ VI. } & 450 & $1 / 2 a$ & Bovine & Semi soft cheese & 2014-01-28 \\
\hline & 6200 & $1 / 2 a$ & Bovine & Semi soft cheese & 2014-06-05 \\
\hline & 6729 & $1 / 2 a$ & Bovine & Semi soft cheese & 2014-06-17 \\
\hline & 7237 & $1 / 2 a$ & Bovine & Semi soft cheese & $2014-06-25$ \\
\hline \multirow{5}{*}{ VII. } & 1103 & $4 b$ & Bovine & Semi soft cheese & 2014-02-11 \\
\hline & 3268 & $4 b$ & Bovine & Semi soft cheese & 2015-04-16 \\
\hline & 3797 & $4 b$ & Bovine & Semi soft cheese & $2015-04-28$ \\
\hline & 9738 & $4 b$ & Sheep & Cheese category not specified & $2015-08-26$ \\
\hline & 2644 & $4 b$ & Sheep & Cheese category not specified & $2015-03-30$ \\
\hline \multirow{3}{*}{ VIII. } & 5898 & $1 / 2 b$ & Bovine & Semi soft cheese & $2014-05-28$ \\
\hline & 6107 & $1 / 2 b$ & - & Food processing environment & 2014-06-03 \\
\hline & 6110 & $1 / 2 b$ & - & Food processing environment & 2014-06-03 \\
\hline
\end{tabular}


environments (farms) with organism and potential crosscontamination of the food. Using more advanced tools (strains serotyping and molecular typing by PFGE), better epidemiologic understanding can be achieved. $46.26 \%$ occurrence of milk associated isolates in a total 147 investigated L.monocytogenes isolates is consistent with the fact that milk and dairy product are one of the most frequent source of listeriosis [4]. The combined analysis of AscI and ApaI patterns of $68 \mathrm{~L}$. monocytogenes strains isolated from dairy product and related environments resulted in strain discrimination into 27 clusters (95\% similarity level). After the selection of clusters with 3 or more profiles, 44 samples were grouped into 8 clusters and one of them (cluster IV) showed the close relationship between samples. All 12 samples from cluster IV were identified to be from one source and one producer. In this case, the subtyping data of L. monocytogenes isolates facilitated finding the source of L. monocytogenes presence in final dairy products and, of course, allowed the producer to optimize the technical and sanitation measures to be taken to ensure hygiene of the food production.

Moreover, all PFGE profiles were sent for curation processing in EURL Lm DB. The majority of them were accepted and involved in the transnational database for sharing with other European countries.

In Slovakia, there have been only a few similar studies focused on solving the secondary contamination problems in food producers using modern typing methods. In one of them, Veghova et al. [11] examined 20 L. monocytogenes strains isolated from sheep milk products and related food processing environments. In contrast with our results, the dominated serogroup in the collection was IIa. The authors identified 14 clusters at a similarity level of $100 \%$ without confirmation of epidemiological clone ECI-ECIII. This high strain similarity suggested the external environment as an origin of contamination [11]. Using molecular tools on national and international levels one can better understand the potential route of disease transmission through the food chains. Databasing system based on PFGE in NRL for Listeria monocytogenes in Slovakia is connected with European databasing system and enables as to compare profiles from all participating countries, not only in the case of disease outbreak, but also for surveillance purposes and epidemiological investigations.

\section{CONCLUSIONS}

L. monocytogenes is a serious foodborne pathogen in human health, and also has important economic influence due to its persistence in food processing environments and potential food product contaminations. In the case of its source and route of contamination detection, suitable analytical method, such as molecular typing, needs to be used. In our surveillance, we referred the potential source and route of milk and dairy products contamination by the identification a cluster (cluster IV) of L.monocytogenes isolates from a specific source (sheep milk, products from sheep milk and related food processing environments) and from one producer. However, another 7 clusters mentioned in Fig. 1 did not show a connection in the context of source and season of contamination or coherence with a specific manufacturer. This example points out the importance and advantages of molecular typing and creating a national database of molecular profiles. Using national databasing systems we were able to search and compare molecular profiles collected through the years. Joining the European databasing system allows the comparison of molecular data with all participating countries and brings benefits during disease outbreak detection, epidemiological investigation and it is also helpful in the case of monitoring and surveillance programmes.

\section{REFERENCES}

1. CDC U, 2010: Outbreak of invasive listeriosis associated with the consumption of hog head cheese - Louisiana, 2010. MMWR Morbidity and Mortality Weekly Report, 60, 401405.

2. CDC U, 2011: Multistate outbreak of listeriosis associated with Jensen Farms cantaloupe - United States, August September 2011. MMWR Morbidity and Mortality Weekly Report, 60, 1357-1358.

3. Choi, M. J., Jackson, K. A., Medu, C., Beal, J., Rigdon, C.E., Cloyd, T. C. et al., 2014: Notes from t8156he field: multistate outbreak of listeriosis linked to soft-ripened cheese - United States, 20815713. MMWR Morbidity and Mortality Weekly Report, 63, 294-295.

4. EFSA (European Food Safety Authority) and ECDC (European Centre for Disease Prevention and Control), 2015: The European Union summary report on trends and sources 
of zoonoses, zoonotic agents and food-borne outbreaks in 2014. EFSA Journal, 13: 4329, 191 pp.

5. Felix, B., Danan, C., VanWalle, I., Lailler, R., Texier, T., Lombard, B., Brisabois, A., Roussel, S., 2014: Building a molecular Listeria monocytogenes database to centralise and share PFGE typing data from food, environmental and animal throughout Europe. J. Microbiol. Methods, 104, 1-8.

6. Graves, L. M., Swaminathan, B., 2001: PulseNet standardized protocol for subtyping Listeria monocytogenes by macrorestriction and pulsed-field gel electrophoresis. Int. J. Food Microbiol., 65, 55-62.

7. Marault, M., Roussel, S., 2011: Molecular subtyping of Listeria monocytogenes using pulsed-field gel electrophoresis. Méthode Anses Maisons-Alfort CEB04, version anglaise, rév. 06, date 28 Février 2011, https://eurl-listeria.anses.fr/en/minisite/ listeria/eurl-lm-house-method-pfge-typing-l-monocytogenes
8. Martin, P., Jacquet, C., Goulet, V., Vaillant, V., De Valk, H., 2006: Pulsed field gel electrophoresis of Listeria monocytogenes strains: the PulseNet Europe Feasibility Study. Foodborne Pathogens and Disease, 3, 303-308.

9. Swaminathan, B., Gerner-Smidt, P., Ng L. K., Lukinmaa, S., Kam, K. M., Rolando, S. et al., 2006: Building PulseNet International: an interconnected system of laboratory networks to facilitate timely public health recognition and response to foodborne diseases. Foodborne Pathogens and Disease, 3, $36-50$.

10. Van Walle, I., 2013: ECDC starts pilot phase for collection of molecular typing data. Euro Surveill., 18, pii: 20357.

11. Véghová, A., Koreňová, J., Minarovičová, J., Drahovská, H., Siekel, P., Kaclíková, E., 2015: Isolation and characterization of Listeria monocytogenes from the environment of three ewes' milk processing factories in Slovakia. Journal of Food and Nutrition Research, 54, 252-259.

Received November 14, 2016

Accepted February 3, 2017 